\title{
Homological Perturbation Theory and the Algebraic Structure of the Antifield-Antibracket Formalism for Gauge Theories
}

\author{
Jean M. L. Fisch ${ }^{1, \star}$ and Marc Henneaux ${ }^{1,2, \star \star}$ \\ ${ }^{1}$ Université Libre de Bruxelles, Faculté des Sciences, Campus Plaine, 231, \\ B-1050 Bruxelles, Belgium \\ ${ }^{2}$ Centro de Estudios Cientificos de Santiago, Casilla 16443, Santiago 9, Chile
}

\begin{abstract}
The algebraic structure of the antifield-antibracket formalism for both reducible and irreducible gauge theories is clarified. This is done by using the methods of Homological Perturbation Theory (HPT). A crucial ingredient of the construction is the Koszul-Tate complex associated with the stationary surface of the classical extremals. The Koszul-Tate differential acts on the antifields and is graded by the antighost number. It provides a resolution of the algebra $\mathscr{A}$ of functions defined on the stationary surface, namely, it is acyclic except at degree zero where its homology group reduces to $\mathscr{A}$. Acyclicity only holds because of the introduction of the ghosts of ghosts and provides an alternative criterion for what is meant by a proper solution of the master equation. The existence of the BRST symmetry follows from the techniques of HPT. The classical Lagrangian BRST cohomology is completely worked out and shown to be isomorphic with the cohomology of the exterior derivative along the gauge orbits on the stationary surface. The algebraic structure of the formalism is identical with the structure of the Hamiltonian BRST construction. The role played there by the constraint surface is played here by the stationary surface. Only elementary quantum questions (general properties of the measure) are addressed.
\end{abstract}

\section{Introduction}

Over the past few years it has become clear that the BRST charge plays a key role in field theory. Recently, the algebraic structure of the classical BRST symmetry has been elucidated in the Hamiltonian formalism for the general case of reducible theories [1]. The crucial point there was the construction of the "Koszul-Tate" differential operator $\delta$ associated with the constraint surface. With this operator at hand, the algebraic properties of the classical BRST charge in the Hamiltonian

* Aspirant du Fonds National de la Recherche Scientifique (Belgium)

$\star \star$ Maître de Recherche du Fonds National de la Recherche Scientifique (Belgium) 
formalism are easily derived and illustrate the methods of Homological Perturbation Theory (HPT) [2, 3]. In light of these methods, one can argue that the algebraic properties of the BRST charge are completely subordinated to the existence of the Koszul-Tate differential ${ }^{1}$.

In the Lagrangian framework, a BRST formalism has also been developed $[9,10]$. This formalism has attracted a great deal of interest lately and has been successfully applied to various models [11]. In this approach, the BRST symmetry is defined in the space of histories of fields and antifields. This space is equipped with a bracket structure (called anti bracket [9]) derived from an odd symplectic 2-form. The (off-shell) classical part of the total Lagrangian must satisfy the master equation, which expresses its nilpotency with respect to the antibracket structure. After a choice for the gauge-fixing fermion $[9,10]$, the solution of the master equation defines both the classical gauge fixed action and the Lagrangian BRST transformation laws.

The symplectic geometries derived from an odd and an even 2-form present some fundamental differences, for instance in the definition of an integration measure. However, we explicitly show in this paper that the construction and the classical properties of the off-shell BRST symmetry in the Lagrangian formalism are algebraic features that can also be completely understood within the framework of HPT. We will actually see that the Lagrangian developments are almost identical in structure to those presented in [1] for the Hamiltonian approach to the classical BRST charge. A crucial role is played again by an auxiliary acyclic complex which determines order by order the solution of the master equation. Once this similarity of structure is understood, the demonstration of the Lagrangian theorems goes without effort. Furthermore, when the equations are written in terms of the appropriate acyclic auxiliary complex, there is very little difference between the reducible and irreducible cases, which can thus be treated simultaneously.

The great similarity between the antifield-antibracket formalism and the Hamiltonian construction of the BRST charge has of course been noticed by many people previously. What is new here is the use of the recent Hamiltonian results of [1] to provide a complete existence proof of solutions to the master equation for arbitrary gauge theories. The analysis of the relationship between gauge invariance and Lagrangian BRST invariance, which falls within the subject of BRST cohomology, appears also to be new.

The paper is organized as follows. In the next section, we first adjust the Lagrangian notations to the one used in [1] so as to make the similarity with the Hamiltonian formalism manifest. Then, in Sect. 3, we show that one can also introduce in the Lagrangian framework an auxiliary nilpotent operator which is

\footnotetext{
${ }^{1}$ The need for higher rank terms in the (Hamiltonian form of the) BRST symmetry of a generic gauge theory was realized in $[4,5]$. That these higher rank terms could only be constructed because of the acyclicity of a certain complex was pointed out in [6] and a step by step proof of the existence of the BRST symmetry was also given there. The fact that the differential complex which appears in the construction had already been considered previously by mathematicians ("Koszul resolution") was indicated in [7]. That these were the ingredients of Homological Perturbation Theory was identified by Stasheff [8]. The point of view of Homological Perturbation Theory turns out to be essential for a proper understanding of reducible theories $[1,8]$
} 
the Koszul-Tate differential [12-14]. This operator acts on the antifields. Requiring it to be acyclic forces the minimal set of variables to be the one introduced in the literature through the proper solution requirement. We then prove in Sect. 4 the existence and uniqueness (modulo a canonical transformation) of the off-shell classical BRST symmetry for reducible theories. This extends the result known for irreducible theories $[15,16]$ to the general case.

Using the nilpotency of the BRST symmetry, we then define in Sect. 5 a classical (Lagrangian) BRST cohomology similar to the one introduced in the canonical formalism [17, 18]. This cohomology is isomorphic with the cohomology of the vertical exterior derivative along the gauge orbits. It plays a useful role in understanding the ambiguity in the Lagrangian quantum measure.

The parallels between the Lagrangian and the Hamiltonian approach to the classical BRST symmetry are so striking that, as we mentioned before, most of the proofs of the theorems we will present are identical to those presented in the Hamiltonian framework in [1]. This is due to the fact that both developments are illustrations of the same algebraic structure (HPT). We therefore mostly refer to that reference for the technical details of the proofs and just stress in this paper the main ideas.

\section{Off Shell Description of Reducible Gauge Theories}

We will slightly alter the notations usually adopted in the literature in order to establish the parallels with the Hamiltonian approach in a more explicit way. Moreover, we present here the off-shell description of gauge theories.

Let us consider an action $S\left(\phi^{a_{0}}\right)$ depending on $m_{0}$ fields $\phi^{a_{0}}$ of Grassmann parity $\varepsilon_{a_{0}}$. We denote the equations of motion derived from $S$ by,

$$
G_{a_{0}}\left(\phi^{b_{0}}\right) \equiv \frac{\partial S}{\partial \phi^{a_{0}}}\left(\phi^{b_{0}}\right)=0, \quad a_{0}=1, \ldots, m_{0} .
$$

This defines a surface in the space of histories which we will call the stationary surface. This surface plays the role of the phase space constraint surface of the Hamiltonian formalism.

The action $S\left(\phi^{a_{0}}\right)$ possesses gauge invariances if there exist non-trivial reducibility relations among the functions $G_{a_{0}}$ which hold everywhere,

$$
\begin{gathered}
G_{a_{0}}\left(\phi^{b_{0}}\right) Z_{a_{1}}^{a_{0}}\left(\phi^{b_{0}}\right)=0, \quad a_{1}=1, \ldots, m_{1}, \\
\varepsilon_{a_{0}}+\varepsilon_{a_{1}}=\varepsilon\left(Z_{a_{1}}^{a_{0}}\right) .
\end{gathered}
$$

We assume that this set of $Z_{a_{1}}^{a_{0}}\left(\phi^{b_{0}}\right)$ completely exhausts on shell the relations among the "stationary constraints,"

$$
G_{a_{0}}\left(\phi^{b_{0}}\right) \lambda^{a_{0}}\left(\phi^{b_{0}}\right)=0 \Leftrightarrow \lambda^{a_{0}}\left(\phi^{b_{0}}\right) \approx Z_{a_{1}}^{a_{0}}\left(\phi^{b_{0}}\right) \lambda^{a_{1}}\left(\phi^{b_{0}}\right) .
$$

The weak equality $\approx$ means equality on the stationary surface.

The gauge invariances may be reducible. In that case, there exists some $Z_{a_{2}}^{a_{1}}\left(\phi^{b_{0}}\right)$ such that,

$$
\begin{gathered}
Z_{a_{1}}^{a_{0}}\left(\phi^{b_{0}}\right) Z_{a_{2}}^{a_{2}}\left(\phi^{b_{0}}\right) \approx 0, \quad a_{2}=1, \ldots, m_{2}, \\
\varepsilon_{a_{1}}+\varepsilon_{a_{2}}=\varepsilon\left(Z_{a_{2}}^{a_{1}}\right)
\end{gathered}
$$


Here also we assume that the reducibility of $Z_{a_{1}}^{a_{0}}$ is completely contained in the $Z_{a_{2}}^{a_{1}}$,

$$
Z_{a_{1}}^{a_{0}}\left(\phi^{b_{0}}\right) \lambda^{a_{1}}\left(\phi^{b_{0}}\right) \approx 0 \Leftrightarrow \lambda^{a_{1}}\left(\phi^{b_{0}}\right) \approx Z_{a_{2}}^{a_{1}}\left(\phi^{b_{0}}\right) \lambda^{a_{2}}\left(\phi^{b_{0}}\right) .
$$

The rank of reducibility may be greater than two, in which case one finds reducibility equations among the reducibility functions $Z_{a_{2}}^{a_{1}}$, etc. .... It is essential that at each stage a complete set of reducibility functions is taken, but this set may be overcomplete. When this occurs, the procedure continues at least to the next stage.

One thus sees that one gets the same basic formal features as for $L^{\text {th }}$ stage reducible Hamiltonian constraint systems $[1,4,5]$. Of course, the meaning of the objects changes. While the Hamiltonian formalism deals with phase space, the present Lagrangian formalism deals with the functional space of Lagrangian histories which is infinite dimensional even in the case of a finite number of degrees of freedom. In the first case, the equations $G_{a_{0}}=0$ refer to the Hamiltonian constraint surface, whereas in the second case, the equations $G_{a_{0}}=0$ (where $a_{0}$ includes the space time coordinates) refer to the Lagrangian stationary surface. Note that this last surface is actually isomorphic to the Hamiltonian constraint surface. Also, a "first-stage" reducible system as defined in [1] would be here an irreducible gauge theory.

In spite of these slight differences, both formalisms possess the same formal structure and necessitate almost identical techniques for deriving the algebraic properties of the classical BRST charge.

We will make the further requirement that the equations of motion as well as the reducibility functions can be locally separated into independent and dependent ones. The independent and dependent equations of motion will be respectively denoted by $G_{A_{0}}=0$ and $G_{\alpha_{0}}=0$, so that $G_{\alpha_{0}}=0$ are consequences of $G_{A_{0}}=0$. We even assume that the rank of $d G_{A_{0}}$ is maximal on the stationary surface. This implies that any function(al) vanishing on $G_{A_{0}}=0$ can be expressed as $\lambda^{A_{0}} G_{A_{0}}$, i.e. that the ideal generated by $G_{A_{0}}$ coincides with the ideal of the function(al)s vanishing on $G_{A_{0}}=0[1,6,10,16]$. This regularity assumption may not hold for all actions, but appears to be essential for the present developments $[1,16]$. If it did not hold, the considerations of this paper (ghost spectrum, BRST invariance versus gauge invariance) would have to be adapted.

This regularity question is more than just of academic interest. For instance, it is not obvious that the regularity property is satisfied by the Siegel model for chiral bosons [19] since some of the field equations only vanish quadratically (and not linearly) on the stationary surface.

With the regularity assumption, one can prove that there always exists locally smooth coordinates such that the equations of motion and the reducibility conditions take the simple form,

$$
\begin{aligned}
G_{a_{0}} & =\left(G_{A_{0}}, G_{\alpha_{0}} \equiv 0\right), \\
Z_{A_{k+1}}^{a_{k}} & =\left(0, \delta_{A_{k+1}}^{\alpha_{k}}\right), \quad k \geqq 0, \\
Z_{\alpha_{k+1}}^{a_{k}} & =(0,0), \quad k \geqq 0,
\end{aligned}
$$

with $G_{A_{0}}$ the first coordinates of the new coordinate system. We used here the notations introduced in the Hamiltonian formalism [1]. 
The regularity assumptions also enable one to replace the weak equations of this section by off-shell statements valid throughout the $\phi^{a_{0}}$-space by adding linear combinations of the $G_{a_{0}}$ to the right-hand side of (2.3), (2.4a) or (2.5). This is permissible since any weakly vanishing function is a combination of the constraints.

Finally, a word of precaution. Since the indices $a_{0}, a_{1}, \ldots$ range also over the spacetime coordinates, there are many delicate functional aspects hidden behind the formulas, [for instance what is the functional class to which the functions such as $\lambda^{a_{0}}$ in (2.3) should belong]. However, these aspects will not be discussed here, for our only purpose is to focus on the algebraic features of the formalism.

\section{The Koszul-Tate Differential Operator $\delta$}

It is well known that the observables of a non-degenerate second order dynamical system are given by the phase space functions. Because a phase space point determines a unique trajectory, one can describe more relativistically the dynamical observables as the functions defined on the stationary surface (2.1) where the equations of motion hold.

Equivalently, one can say that the observables are function(al)s $F\left(\phi^{a_{0}}\right)$ defined in the space of all possible histories, provided one identifies two function(al)s which coincide on shell,

$$
F\left(\phi^{a_{0}}\right) \sim F^{\prime}\left(\phi^{a_{0}}\right) \text { iff } F-F^{\prime} \approx 0 .
$$

In the case of gauge theories, one can still adopt the same description, but one must impose in addition that the functions be (weakly) gauge invariant,

$$
\frac{\delta F}{\partial \phi^{a_{0}}}\left(\phi^{b_{0}}\right) Z_{a_{1}}^{a_{0}}\left(\phi^{b_{0}}\right) \approx 0
$$

We analyse here how to implement the equivalence relation (3.1). The question of gauge invariance (3.2) will be examined in Sect. 5 .

The functions $F\left(\phi^{a_{0}}\right)$ form an algebra $R$ because they can be multiplied among each other. The functions which vanish on shell form an ideal $I$ of this algebra since if $A\left(\phi^{a_{0}}\right) \approx 0$ (i.e. $A \in I$ ), then $(A F)\left(\phi^{a_{0}}\right) \approx 0$, even if $F \approx 0$. As we have seen, this ideal is the ideal generated by $G_{a_{0}}$, i.e. its elements are of the form $G_{a_{0}} \lambda^{a_{0}}$ (regularity condition). The algebra $\mathscr{A}$ of functions on the stationary surface is isomorphic with the quotient $R / I$.

Now, one can give a homological interpretation of $R / I$. This reformulation of the equivalence relation (3.1) turns out to be a cornerstone of the BRST construction and goes as follows.

Introduce one antifield $\phi_{a_{0}}^{*}$ of opposite statistics for each field $\phi^{a_{0}}$ and consider the differential complex generated by $\phi^{a_{0}}$ and $\phi_{a_{0}}^{*}$, graded by the antighost number,

$$
\operatorname{antigh}\left(\phi^{a_{0}}\right)=0, \operatorname{antigh}\left(\phi_{a_{0}}^{*}\right)=1 .
$$

The differential is defined so that the functions $G_{a_{0}}$ are exact, i.e.,

$$
\delta \phi_{a_{0}}^{*}=G_{a_{0}}
$$


and so that it annihilates the fields $\phi^{a_{0}}$ (as well as all the extra fields $\phi^{a_{k}}$ introduced later which also have antighost number zero). One extends $\delta$ to an arbitrary element of the complex by means of the graded Leibnitz rule, with $\delta$ acting from the left. Note that in the Hamiltonian formalism [1], $\delta$ was chosen to act as a right derivative.

By construction, $\delta$ is nilpotent since $\delta G_{a_{0}}\left(\phi^{b_{0}}\right)=0$. Furthermore, its homology at order zero is easily computed. Any function $F\left(\phi^{a_{0}}\right)$ is closed, while the exact functions are given by $\delta\left(\phi_{a_{0}}^{*} \lambda^{a_{0}}\right)=G_{a_{0}} \lambda^{a_{0}}$, i.e., belong to the ideal $I$. This implies

$$
(\operatorname{Ker} \delta / \operatorname{Im} \delta)_{0} \equiv H_{0}(\delta)=R / I \text {. }
$$

Hence, the algebra $\mathscr{A}$ of functions defined on the stationary surface can be described as the zero ${ }^{\text {th }}$ homological class of the differential $\delta$.

What are the higher order homological classes? In the absence of gauge invariance, the functions $G_{a_{0}}$ would be independent and one could then show that the cohomological classes at antighost number $p>0$ all reduce to zero $[6,12,14]$. However, acyclicity at $p>0$ does not hold for a theory with gauge invariance, for one can find non-trivial cycles already at antighost number one. Because the reducibility functions $Z_{a_{1}}^{a_{0}}$ exhaust all non-trivial relations among the $G_{a_{0}}$,s, a basis of these non-trivial cycles is given by

$$
\phi_{a_{0}}^{*} Z_{a_{1}}^{a_{0}}, \delta\left(\phi_{a_{0}}^{*} Z_{a_{1}}^{a_{0}}\right)=G_{a_{0}} Z_{a_{1}}^{a_{0}}=0 .
$$


to vanish on shell.

As it will become explicit in the next sections, acyclicity of $\delta$ at antighost number $\neq 0^{2}$ is a much desired property, which plays a key role in the BRST construction. It is not clear that appropriate solutions of the master equation would exist if that property were to be given up. So, it appears necessary to recover acyclicity. This can be done following Tate [13], by adding extra variables, which are just the extra antifields.

These extra antifields $\phi_{a_{1}}^{*}$ are introduced so as to kill the cycles (3.6), i.e., one defines

$$
\begin{gathered}
\delta \phi_{a_{1}}^{*}=\phi_{a_{0}}^{*} Z_{a_{1}}^{a_{0}}, \\
\operatorname{antigh}\left(\phi_{a_{1}}^{*}\right)=2, \quad \varepsilon\left(\phi_{a_{1}}^{*}\right)=\varepsilon_{a_{1}}+1 .
\end{gathered}
$$

One has clearly $\delta^{2} \phi_{a_{1}}^{*}=0$ (since $\phi_{a_{0}}^{*} Z_{a_{1}}^{a_{0}}$ is closed), and $\phi_{a_{0}}^{*} Z_{a_{1}}^{a_{0}}$ has become exact by construction. Hence, after the antifields $\phi_{a_{1}}^{*}$ have been added, the first homology group $H^{1}(\delta)$ of $\delta$ is trivial, $H^{1}(\delta)=0$.

The procedure must be continued if there are further reducibility equations among the $Z$ 's, for one would then find $H^{2}(\delta) \neq 0$, etc.... In order to recover acyclicity at all orders, one must introduce further antifields $\phi_{a_{2}}^{*}, \phi_{a_{3}}^{*}, \ldots$ at each reducibility stage, with antigh $\left(\phi_{a_{k}}^{*}\right)=k+1, \varepsilon\left(\phi_{a_{k}}^{*}\right)=\varepsilon_{a_{k}}+1$ and define

$$
\delta \phi_{a_{k}}^{*}=\phi_{a_{k-1}}^{*} Z_{a_{k}}^{a_{k-1}}+M_{a_{k}}\left(\phi_{a_{0}}^{*}, \ldots, \phi_{a_{k-2}}^{*}\right), \quad k \geqq 1 .
$$

The functions $M_{a_{k}}$ are determined so that $\delta^{2} \phi_{a_{k}}^{*}=0$ and arise when $Z_{a_{k-1}}^{a_{k-2}} Z_{a_{k}}^{a_{k-1}}$ only vanish on-shell.

\footnotetext{
${ }^{2}$ From now on, "acyclicity" will always mean "acyclicity except at order zero"
} 
The precise determination of $M_{a_{k}}$ and the explicit checking that the introduction of the extra antifields according to (3.8) removes all the non-trivial cycles proceeds as in the Hamiltonian formalism. So, we refer to [1] for the proofs.

The complex generated by the antifields, with the differential $\delta$ defined by (3.4), (3.7), (3.8) is called the Koszul-Tate complex. It provides a resolution of the quotient algebra $\mathscr{A} \equiv R / I$, in the sense that $H^{0}(\delta)=\mathscr{A}, H^{i}(\delta)=0, i \neq 0$. The differential $\delta$ turns out to be equivalent for irreducible gauge theories to the operators $W$ and $\Omega$ respectively introduced in [15] and [16].

The demand that the differential $\delta$ be acyclic automatically forces the antifield spectrum to be just the correct minimal set of [10]. Consequently, it also correctly determines the spectrum of the conjugate ghosts of ghosts.

This correct ghost spectrum was arrived at [10] in a different manner, by requiring that $S$ be a proper solution of the master equation. By this, it was meant that the $2 N \times 2 N$ Hessian matrix of the second derivatives of $S$ with respect to both the fields and the antifields, which is on-shell nilpotent, should be of maximal possible rank $N$.

That both requirements are equivalent is not surprising once it is realized (i) that a nilpotent $2 N \times 2 N$ matrix is of maximum $\operatorname{rank} N$ if and only if it defines an "acyclic" operator ${ }^{3}$; and (ii) that the Hessian matrix and $\delta$ are both built out of the same reducibility functions $Z_{a_{k+1}}^{a_{k}}$.

Although the two criteria are equivalent and yield the same minimal spectrum of fields and antifields, it should be noted that the proper solution requirement can only be formulated on shell where the Hessian matrix is nilpotent, while acyclicity of $\delta$ is an off-shell statement.

\section{Existence and Uniqueness of the Proper Solution to the Master Equation}

As it has been extensively shown in $[9,10]$, the classical part $S$ of the total Lagrangian plays also the role of BRST generator when acting through the antibracket. It is an even element of the Grassmann algebra with zero ghost number and is a proper solution of the master equation,

$$
(S, S)=0 \text {. }
$$

We adopt here the ghost number convention given in [10], $\operatorname{gh}\left(\phi_{a_{k}}^{*}\right)=-k-1$ $=-\operatorname{antigh}\left(\phi_{a_{k}}^{*}\right)$ and $\operatorname{gh}\left(\phi^{a_{k}}\right)=k, \operatorname{antigh}\left(\phi^{a_{k}}\right)=0$. The ghost number refers in these equations to the total ghost number.

In order to investigate the master equation, one expands $S$ with respect to the grading of the Koszul-Tate differential $\delta$, i.e. with respect to the antighost number,

$$
S=\sum_{n \geqq 0} \stackrel{(n)}{S}, \quad \operatorname{antigh}(\stackrel{(n)}{S})=n .
$$

\footnotetext{
${ }^{3}$ Let $R: V \rightarrow V$ be an endomorphism of a vector space $V$ of dimension $2 N$. Assume that $R$ is nilpotent, $R^{2}=0$. If the equation $R v=0$ implies $v=R u$, for some $u$, i.e., if $\operatorname{Ker} R / \operatorname{Im} R=\{0\}$, then $R$ is of rank $N$. This is the maximum rank for a nilpotent matrix in $V$. The proof of this statement can be easily given in a Jordan canonical basis for $R$. The condition $\operatorname{ker} R=\operatorname{Im} R$ implies that $R$ contains only two-dimensional Jordan blocks $\left(\begin{array}{ll}0 & 1 \\ 0 & 0\end{array}\right)$, which are of rank one
} 
This significantly simplifies the structure of the equations derived from the master equation. Note that this has been indirectly adopted in $[15,16]$ for irreducible gauge theories, since there the ghost sector is restricted to the single $\phi^{a_{1}}$ set.

In terms of this antighost number expansion, the master equation gives rise to the infinite set of equations,

$$
\begin{gathered}
\bar{\delta}^{(n+1)} S+\stackrel{(n)}{S}(\stackrel{(0)}{S}, \ldots, \stackrel{(n)}{S})=0, \quad n \geqq 0, \\
\bar{\delta}(\cdot)=\sum_{k=0}^{n} \frac{\overbrace{}^{(k)} S}{\partial \phi^{a_{k}}} \frac{\vec{\partial}(\cdot)}{\partial \phi_{a_{k}}^{*}},
\end{gathered}
$$

where $\stackrel{(0)}{D}$ identically vanishes and $\stackrel{(n)}{D}$ involves antibrackets of $\stackrel{(0)}{S}, \ldots, \stackrel{(n)}{S}$ up to order $n$. Its explicit form will not be needed here. We will see below that $\bar{\delta}$ in (4.3) coincides with the Koszul-Tate differential of the precious section. So, (4.3) can be investigated by means of homological techniques.

The fact that the master equation reduces to the set of equations (4.3a) with $\bar{\delta}=$ Koszul-Tate is a non-trivial result in the reducible case. This structure would not be directly apparent in general if we had expanded $S$ in powers of the fields or in powers of the antifields, with equal weight given to each field or antifield in the expansion. The crucial step in the analysis of the master equation is to make explicit the appearance of the Koszul-Tate differential, and this is manifestly achieved if we expand $S$ according to the antighost number.

Note that there are in (4.3) some slight differences with the Hamiltonian approach. In the Lagrangian framework, $\stackrel{(0)}{D}$ identically vanishes. This is of course expected since the original classical action $S\left(\phi^{a_{0}}\right)$ itself defines a solution to the master equation (which is not proper if the action has a gauge invariance $[9,10]$ ). In the Hamiltonian BRST formalism, the weak vanishing of $\stackrel{(0)}{D}$ is a consistency requirement for the BRST charge to exist. This requirement expresses nothing else than the fact that, in the Hamiltonian formalism, the standard BRST charge can only be directly defined when the constraints are first class, i.e. correspond to a gauge invariance.

The proper boundary conditions to be imposed on $S$ in order for $S$ to be a proper solution [10] take in our notation the form,

$$
\begin{gathered}
\stackrel{(0)}{S}=S\left(\phi^{a_{0}}\right), \\
\frac{\vec{\partial}}{\partial \phi_{a_{k-1}}^{*}} \frac{\partial S^{(k)}}{\partial \phi^{a_{k}}}=Z_{a_{k}}^{a_{k-1}}\left(\phi^{k_{0}}\right), \quad k \geqq 1 .
\end{gathered}
$$

These boundary conditions can be given a physical motivation a posteriori when we investigate the BRST cohomology in the next section. Let us simply remark here that it is only with (4.4b) that the operator $\bar{\delta}$ of equation (4.3b) reduces to the acyclic Koszul-Tate differential of the previous section.

Given the boundary conditions (4.4) and the form (4.3) of the master equation, one just needs to apply the algebraic techniques of HPT along the same lines as in the Hamiltonian approach [1] to prove the following fundamental theorem. 
Theorem 4.1. There exists a classical solution $S$ of the master equation obeying the boundary conditions (4.4). Moreover, this solution is unique up to a canonical transformation (in the antibracket sense).

This is the extansion to the general case of the theorem proven in [15] and [16] for the particular case of irreducible gauge theories.

Let us sketch the general proof which goes by induction. Assume that $\stackrel{(0)}{S}, \ldots, \stackrel{(n)}{S}$ have been constructed up to order $n$, in accordance to the boundary conditions (4.4). The first step then is to observe that $\bar{\delta}$ can indeed be identified with the Koszul-Tate differential up to antighost number $n+1$. This follows from the boundary conditions (4.4b), which imply that $\bar{\delta}$ and $\delta$ coincide to leading order, and from the fact that $\bar{\delta}$ and $\delta$ are nilpotent.

Because $\delta=\bar{\delta}$ is not only nilpotent but also acyclic, the existence of $S$ amounts to the proof of $\delta \stackrel{(n)}{D}=0$. Two proofs of this identity can be given and we outline them both here (the exact relationship between these two proofs has not been entirely worked out).

The first proof is purely algebraic and is based on the Jacobi identity $\left(\left(S_{n}, S_{n}\right), S_{n}\right) \equiv 0$ for the part $S_{n} \equiv \sum_{m \leqq n} \stackrel{(m)}{S}_{m}$ of $S$ that has already been constructed [16]. Since $S_{n}$ is nilpotent up to the order $n$, this identity just reduces to $\delta \stackrel{(n)}{D}=0$ at leading antighost order as required. Note that the identity $\delta \stackrel{(n)}{D}=0$ on the Lagrangian structure functions $\stackrel{(n)}{S}$ has been first derived in the irreducible case by a different method in [20]. Note also that the same algebraic proof can be adapted to the Hamiltonian formalism (when developing in components, one then recovers the lengthy expressions of the appendix of the second reference in [6]).

The second proof is more geometrical and extends the approach adopted in [15] for irreducible gauge theories. Because $\delta \stackrel{(n)}{D}=0$ is an algebraic statement, it is sufficient to check it locally. This can then most easily be done by going over to the local coordinates in which the reducibility coefficients (and consequently $\stackrel{(0)}{S}, \ldots, \stackrel{(n)}{S}$ ) take a simple form. This second proof is the exact pendant of the existence proof of the BRST charge in the Hamiltonian formalism given in [1].

The proof that $S$ is unique up to a canonical transformation [15] then goes along the same lines as in the Hamiltonian case [1].

\section{Classical Lagrangian BRST Cohomology}

In the previous sections, we arrived at the correct ghost spectrum by requiring acyclicity of the Koszul-Tate differential. In this section, we are going to give a more physical motivation for the introduction of the ghosts and the ghost of ghosts.

A key feature in the BRST formalism is that global BRST invariance replaces gauge invariance as the symmetry on which the gauge-fixed Lagrangian is built. One therefore expects that BRST invariance guarantees gauge invariance. This is 
achieved by introducing the right ghost spectrum to exactly kill the unphysical degrees of freedom.

On these grounds, BRST invariant functions (with zero ghost number) should correspond to gauge invariant functions. Due to the Jacobi identity, BRST-exact functions $(S, X)$ with $\varepsilon(X)=1$ and $\operatorname{gh}(X)=-1$ trivially define BRST-invariant functions. However this special class of BRST-invariant functions does not play a role at the quantum level. Indeed, their quantum average on the gauge fixing surface can formally be shown to vanish. As a result, the quantum average of physically relevant (BRST-invariant) functions is effectively defined on the cohomology space $(\operatorname{Ker} S / \operatorname{Im} S)^{0}$. From this physical argument, the cohomology space $(\operatorname{Ker} S / \operatorname{Im} S)^{0}$ should be isomorphic to the space of (physically inequivalent) gauge invariant functions of the original $\phi^{a_{0}}$ fields. The ghost spectrum should therefore be chosen such that this physical requirement holds.

One can provide a more geometrical description of gauge invariant functions in the $\phi^{a_{0}}$-space. On the stationary surface, the gauge transformations are integrable. The gauge orbits that they generate define vertical directions and one can introduce an exterior derivative acting on the vertical forms. This exterior derivative measures the variation of a vertical form along the gauge orbits (the vertical directions). It does not provide any information on the change induced by going from one gauge orbit to another.

In this description, physically inequivalent gauge invariant functions are just the $d$-closed zero forms and are described by the cohomology space $(\operatorname{Ker} d / \operatorname{Im} d)^{0}$.

Therefore, the physical requirement that the BRST cohomology class $(\operatorname{Ker} S / \operatorname{Im} S)^{0}$ should be isomorphic to the physically inequivalent gauge invariant functions can be summarized as $(\operatorname{Ker} S / \operatorname{Im} S)^{0} \simeq(\operatorname{Ker} d / \operatorname{Im} d)^{0}$.

This turns out to be the case. One can in fact prove more, namely that $(\operatorname{Ker} S / \operatorname{Im} S)^{p} \simeq(\operatorname{Ker} d / \operatorname{Im} d)^{p}$, where $p \geqq 0$. For the BRST cohomology at negative ghost number, the cohomology spaces are found to be trivial, $(\operatorname{Ker} S / \operatorname{Im} S)^{p}=0$ for $p<0$.

The proof of these results follows the same lines as in the Hamiltonian approach $[1,17,18]$, and we will not reproduce them here. Let us simply provide here an example that these proofs crucially depend on the spectrum of ghosts introduced in the previous sections.

Consider the following reducible set of gauge invariances $\delta_{1} \phi=\varepsilon^{1}, \delta_{2} \phi=0 \cdot \varepsilon^{2}$, and $\delta_{3} \phi=0 \cdot \varepsilon^{3}$ of the action $\stackrel{(0)}{S}=0$. The space of physically inequivalent gauge invariant functions is just given by the constant functions. Without introducing any ghosts, the BRST cohomology, given by $(\operatorname{Ker} \stackrel{(0)}{S} / \operatorname{Im} \stackrel{(0)}{S})$ is isomorphic with the ring of $\phi$-dependent functions and would not reproduce the physically desired result. Moreover, introducing the ghosts $\eta^{i}(\mathrm{i}=1,2,3)$ without the ghosts for ghosts is also not enough. In this case, $S$ reads as $S=\phi^{*} \eta^{1}$ and the BRST cohomology space $(\operatorname{Ker} S / \operatorname{Im} S)^{0}$ contains, besides the constants, ghost-dependent elements, for instance $\eta_{2}^{*} \eta^{2} \eta^{3}$. The reader can check on this example that one recovers the isomorphism between $(\operatorname{Ker} S / \operatorname{Im} S)^{0}$ and $(\operatorname{Ker} d / \operatorname{Im} d)^{0}$ only after one has introduced the whole generation of ghosts which makes the Koszul-Tate differential acyclic. 
So far, we only considered the BRST cohomology associated with minimal proper solutions of the master equation. In the path integral based on the antifieldantibracket formalism, however, non-minimal solutions are also of interest ${ }^{4}$. These non-minimal solutions possess the same BRST cohomology as the one derived from the underlying minimal solution. Indeed, as a consequence of the Künneth formula, the cohomology of the non-minimal solution is the tensor product of the cohomology of the minimal one by the cohomology carried by the additional nonminimal terms. This last cohomology being trivial, it is therefore sufficient for cohomological purposes to consider minimal solutions.

An application of the BRST cohomology concerns the problem of the quantum measure, which must obey the equations [9],

$$
\begin{gathered}
\left(W_{1}, S\right)=i \Delta S, \\
\left(W_{n+1}, S\right)=i \Delta W_{n}-\frac{1}{2} \sum_{m=1}^{n}\left(W_{m}, W_{n+1-m}\right), \quad n \geqq 1 .
\end{gathered}
$$

These are formal equations and need prescriptions for dealing with the hidden $\delta(0)$ factors. These prescription questions fall outside the scope of the present paper. However, once $W_{1}, W_{2}, \ldots, W_{n}$ are given, $W_{n+1}$ is determined by an inhomogeneous linear equation $(5.2 \mathrm{~b})$. The general solution to this equation is given by a particular solution plus the general solution to the homogeneous equation $\left(W_{n+1}, S\right)=0$. From the discussion of this section, the general solution to this homogeneous equation is given by the gauge invariant functions modulo an arbitrary $S$-exact term $\left(S, X_{n+1}\right)$. This last arbitrary term can be eliminated from the path integral by performing an $\hbar$-dependent change of variables [21].

The conclusion is that the quantum measure is determined by (5.2) up to an arbitrary function of the gauge invariant variables. To present it differently, BRST invariance fixes the measure in the gauge sector, but further requirements seem to be needed for fixing the measure in the gauge invariant (i.e. BRST invariant) sector.

\section{Remarks and Conclusions}

In this paper, we have shown that the solution of the master equation in the antifield-antibracket formalism can be constructed following the methods of Homological Perturbation Theory. The construction is based on the Koszul-Tate complex, which provides a resolution of the quotient algebra of functions on the stationary surface. We have also indicated how BRST invariance in the Lagrangian formalism incorporates gauge invariance.

The algebraic similarities between the Lagrangian and Hamiltonian approaches are striking and point to the fact that a further analysis of the detailed correspondence between the two descriptions is likely to be interesting. The

\footnotetext{
${ }^{4}$ The reason to this lies in the wish to define the gauge fixing surface for the path integral through a gauge fermion $\psi$. However, the gauge fermion should be constructed out of the fields and at the same time be of ghost number minus one. This forces one to introduce auxiliary fields (and antifields) with negative ghost number. The precise prescriptions associated with these developments can be found in [10]
} 
comparison, however, has only been carried through for specific systems and further work is clearly needed (for a recent string example, see [22]). This question is presently under investigation [23].

Finally, we note that the theorems on the existence of the nilpotent action $S$ and on the BRST cohomology still hold in the case of infinitely reducible gauge systems.

This is because the formalism is expanded according to the antighost number $p$. For any definite value of $p$, only a finite number of generations of ghosts are involved in the calculation, so that one never has to deal with infinite series.

It is possible to exhibit, however, ill-defined expressions for well-defined quantities. To display a simple example, let us go over to the Hamiltonian formalism where the same features hold. The number of degrees of freedom can be written in that formalism as $n-m_{0}+m_{1}-m_{2}+\ldots$, an expression which diverges in the infinitely reducible case. How the series should be interpreted is indicated by the BRST cohomology, which groups the variables into mutually cancelling pairs and which thus tells one where the parentheses should be placed in the sum.

To give concrete models in which this feature is displayed, consider the following three systems with two canonically conjugate pairs $\left(x^{1}, p_{1}\right)$ and $\left(x^{2}, p_{2}\right)$. The constraints are

$$
\text { (I) }\left(G_{1}, G_{2}\right) \equiv(0,0) \approx 0
$$

in the first case,

$$
\text { (II) }\left(G_{1}, G_{2}\right) \equiv\left(p_{1}, p_{2}\right) \approx 0
$$

in the second case, and

$$
\text { (III) }\left(G_{1}, G_{2}\right) \equiv\left(p_{1}, 0\right) \approx 0
$$

in the last case. These systems can be viewed as infinitely reducible if one takes as reducibility matrices the following $2 \times 2$ matrices

$$
\begin{aligned}
\text { (I) } Z_{a_{2 k+1}}^{a_{2 k}}=\left(\begin{array}{ll}
1 & 0 \\
0 & 1
\end{array}\right), & Z_{a_{2 k+2}}^{a_{2 k+1}}=\left(\begin{array}{ll}
0 & 0 \\
0 & 0
\end{array}\right), \\
\text { (II) } Z_{a_{2 k+1}}^{a_{2 k}}=\left(\begin{array}{ll}
0 & 0 \\
0 & 0
\end{array}\right), & Z_{a_{2 k+2}}^{a_{2 k+1}}=\left(\begin{array}{ll}
1 & 0 \\
0 & 1
\end{array}\right), \quad k \geqq 0, \\
\text { (III) } Z_{a_{k+1}}^{a_{k}}=\left(\begin{array}{ll}
0 & 0 \\
1 & 0
\end{array}\right), & k \geqq 0 .
\end{aligned}
$$

The number of degrees of freedom can be formally written in each case as $2-2+2=2+\ldots$. In the system (I), this sum stands for $2-(2-2)-(2-2)-\ldots=2$ since this is how the variables cancel in pairs. In the second case, one finds $(2-2)$ $+(2-2)+\ldots=0$. Finally, in the third case, one has a mixture of (I) and (II), i.e., the sum equals $[1-(1-1)-(1-1)-\ldots]+[(1-1)+(1-1)+\ldots]=1$. Another way to arrive at this last result is to observe that the reducibility matrices are identical at each generation, and thus the sum should be rewritable as $s=2-(2-(2-(2-\ldots$, i.e. $s=2-s$ and so $s=1$. 
These values, of course, correspond to the true number of degrees of freedom of the respective models.

Acknowledgements. We are grateful to Jim Stasheff and Claudio Teitelboim for illuminating discussions. This work has been supported in part by NSF Grant PHY-8600334 to the University of Texas at Austin.

\section{References}

1. Fisch, J., Henneaux, M., Stasheff, J., Teitelboim, C.: Commun. Math. Phys. 120, 379 (1989)

2. Hirsch, G.: Bull. Soc. Math. Belg. 6, 79 (1953) Stasheff, J.D.: Trans. Am. Math. Soc. 108, 215, 293 (1963)

3. Gugenheim, V.K.A.M.: J. Pure Appl. Alg. 25, 197 (1982)

Gugenheim, V.K.A.M., May, J.P.: Mem. AMS 142 (1974)

Gugenheim, V.K.A.M., Stasheff, J.D.: Bull. Soc. Math. Belg. 38, 237 (1986)

Lambe, L., Stasheff, J.: Manus. Math. 58, 363 (1987)

4. Fradkin, E.S., Fradkina, T.E.: Phys. Lett. 72 B, 343 (1978)

5. Batalin, I.A., Fradkin, E.S.: Phys. Lett. 122 B, 157 (1983)

6. Henneaux, M.: Thèse d'Agrégation (Bruxelles 1984); Phys. Rep. 126 C, 1 (1985)

Contribution in: Quantum mechanics of Fundamental Systems 1. Teitelboim, C. (ed.), New York: Plenum Press 1988, p. 117

7. McMullan, D.: J. Math. Phys. 23, 428 (1987)

Browning, A.D., McMullan, D.: J. Math. Phys. 28, 438 (1987)

8. Stasheff, J.D.: Suppl. Rend. Circ. Mat. Palermo II, 16, 239 (1987); Bull. Am. Math. Soc. 19, 287 (1988); Homological reduction of constrained algebras. North Carolina preprint (August 1988)

9. Batalin, I.A., Vilkovisky, G.A.: Phys. Lett. 102 B, 27 (1981)

10. Batalin, I.A., Vilkovisky, G.A.: Phys. Rev. D 28, 2567 (1983); Phys. Rev. D 30, 508 (1984)

11. Bochicchio, M.: Phys. Lett. 193 B, 31 (1987); Phys. Lett. 188 B, 330 (1987)

de Alwis, S.P., Grisara, M.T., Mezincescu, L.: Nucl. Phys. B 303, 57 (1988)

Diaz, A.H.: Phys. Lett. 203 B, 408 (1988)

Baulieu, L., Bergshoeff, E., Sezgin, E.: Nucl. Phys. B 307, 348 (1988)

Batlle, C., Gomis, J.: Phys. Rev. D 38, 1169 (1988)

Batlle, C.: Teories de camps de cordes. Ph. D. Thesis (Barcelona, 1988)

Labastida, J.M.F., Pernici, M., Witten, E.: Nucl. Phys. B 310, 611 (1988)

12. Koszul, J.L.: Bull. Soc. Math. France 78, 5 (1950)

13. Tate, J.: Illinois J. Math. 1, 14 (1957)

14. Borel, A.: Ann. Math. 57, 115 (1953)

15. Voronov, B.L., Tyutin, I.V.: Theor. Math. Phys. 50, 218 (1982)

16. Batalin, I.A., Vilkovisky, G.A.: J. Math. Phys. 26, 172 (1985)

17. Henneaux, M., Teitelboim, C.: Commun. Math. Phys. 115, 213 (1988)

18. Dubois-Violette, M.: Ann. Inst. Fourier 37, 45 (1987)

19. Siegel, W.: Nucl. Phys. B 238, 307 (1984)

20. de Wit, B., van Holten, J.W.: Phys. Lett. 79 B, 389 (1979)

21. Dixon, J.A.: Nucl. Phys. B 99, 420 (1975)

22. Del Duca, V., Magnea, L., van Nieuwenhuizen, P.: Int. J. Mod. Phys. A 3, 1081 (1988)

23. Batlle, C., Gomis, J., Paris, J., Roca, J.: Phys. Lett. 224 B, 288 (1989)

Fisch, J.M.L., Henneaux, M.: Phys. Lett. 226 B, 80 (1989)

Siegel, W.: Int. J. Mod. Phys. A 4, 3951 (1989) 
24. Henneaux, M.: Lectures on the antifield BRST formalism for gauge theories, ULB preprint ULB TH2/89-07 (September, 1989), to appear in Proceedings of the 1989 GIFT Meeting (North Holland, Amsterdam 1990) and In: Quantum Mechanics of Fundamental Systems III, Teitelboim, C., Zanelli, J. (eds.). New York: Plenum Press 1990

25. Fisch, J.M.L.: On the antibracket-antifield BRST formalism and its applications, ULB preprint ULB TH2/90-01 (Ph. D. Thesis, in preparation)

Communicated by L. Alvarenz-Gaumé

Received Fabruary 13, 1989

Note added in proof. Strictly speaking, one can distinguish between two BRST cohomologies, the antibracket BRST cohomology which has been investigated here and the effective BRST cohomology. The latter is based on the BRST symmetry of the gauge fixed action. It is defined as the set of operators which are BRST invariant on shell but which are not equal to the BRST variation of some operator modulo equations of motion of $S_{\Psi}$. One can prove that these two cohomologies are isomorphic if the action $S_{\Psi}$ is gauge fixed [24, 25]. 